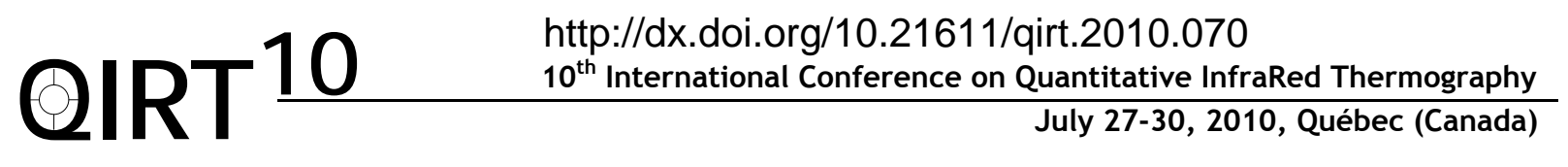

\section{INFRARED PHOTO-THERMOGRAPHY FOR STANDOFF DETECTION OF EXPLOSIVE SUBSTANCES AT DISTANCES UP TO 150 METERS}

\author{
C. Kumar N. Patel \\ Pranalytica, Inc., 1101 Colorado Avenue, Santa Monica, CA 90401 (USA) \\ and
}

Department of Physics \& Astronomy, University of California, Los Angeles, CA 90095

\begin{abstract}
The availability of relatively high power infrared lasers have made it possible to exploit infrared photothermography as a powerful tool in standoff detection of trace amounts of wavelength selective absorbing materials. We have successfully detected and identified trace quantities of explosives at standoff distances up to 150 meters with high sensitivity (SNR 70) and high selectivity using step tunable $\mathrm{CO}_{2}$ lasers. The technique involves illuminating the target object with laser radiation at a wavelength that is strongly absorbed by the target. The resulting temperature rise is observed by remotely monitoring the increased blackbody radiation from the sample. An unambiguous determination of the target, TNT, in soil samples collected from explosives test site in China Lake Naval Air Weapons Station is achieved through the use of a tunable $\mathrm{CO}_{2}$ laser that scans over the absorption fingerprint of the target explosives. Soil samples that are known to be uncontaminated by explosive residues are used to confirm the wavelength selective identification of contaminated soil samples. The theoretical analysis supports the observation and indicates that with optimized detectors and data processing algorithms, the measurement capability can be improved significantly, permitting rapid standoff detection of explosives at distances approaching $1 \mathrm{~km}$. The detection sensitivity varies as $\mathrm{R}^{-2}$ and thus with the availability of high power, room temperature, tunable mid wave infrared (MWIR) and long wave infrared (LWIR) quantum cascade lasers, this technology may play an important role in screening personnel and their belongings at short distance, such as airports, for detecting and identifying explosives material residue on persons as well as identifying improvised explosive devices from safe distances. I will also present preliminary data on the standoff detection of dangerous gaseous materials at distances up to 250 meters.
\end{abstract}

